\title{
Genetics and management of locally advanced carcinomas of the head and neck: role of altered fractionation radiotherapy
}

Future Science $\mathrm{OA}$

\author{
Francesco Perri*,1, Franco Ionna², Paolo Muto ${ }^{3}$, Massimiliano Di Marzo ${ }^{4}$, Francesco \\ Caponigro ${ }^{1}$, Francesco Longo ${ }^{2}$, Giuseppina Della Vittoria Scarpati ${ }^{5}$, Giuseppe Di Lorenzo ${ }^{6}$, \\ Mario Giuliano $0^{6,7}$ \& Raffaele Solla ${ }^{8}$ \\ ${ }^{1}$ Head \& Neck, Soft Tissue Sarcoma Medical Oncology Department, National Tumor Institute of Naples, IRCCS G Pascale, Napoli, \\ Naples, Italy \\ ${ }^{2}$ Otolaryngology Unit, Department of Head \& Neck, Soft Tissue Sarcoma \& Thyroid Cancer, National Tumor Institute of Naples, \\ IRCCS G Pascale, Napoli, Naples, Italy \\ ${ }^{3}$ Radiation Therapy Department, National Tumor Institute of Naples, IRCCS G Pascale, Napoli, Naples, Italy \\ ${ }^{4}$ Head \& Neck - Soft Tissue Sarcoma Unit, IRCCS G Pascale, Naples, Italy \\ ${ }^{5}$ Medical Oncology Unit, POC SS Annunziata, Taranto, Italy \\ ${ }^{6}$ Department of Clinical Medicine \& Surgery, University Federico II, Naples, Italy \\ ${ }^{7}$ Department of Medical Oncology, Lester \& Sue Smith Breast Cancer Center, Baylor College of Medicine, Houston, TX, USA \\ ${ }^{8}$ Department of Radiation Oncology, Italian National Research Council, Institute of Biostructure \& Bioimaging, Naples, Italy \\ *Author for correspondence: Tel.: +39 348914 5086; francesco.perri80@alice.it
}

Squamous cell carcinoma of the head and neck (SCCHN) accounts for $5-7 \%$ of all malignancies. About $60 \%$ of newly diagnosed SCCHN are detected as locally advanced disease. Chemoradiation is a standard option and response rate to it is variable. Recently, a genetic classification of SCCHN has been proposed by Chung et al., who categorized all SCCHN into four subtypes. The basal-like variant is characterized by high expression of EGFR. Literature data suggest higher efficacy of accelerated and/or hyperfractionated radiotherapy, if compared with conventional radiotherapy in the subgroup of patients with high EGFR expression. In this review, we will describe the genetic factors able to guide treatment choice, with a focus on EGFR expression.

Lay abstract: Management of head and neck squamous cell carcinomas is very challenging, with malignancies often strongly dampening the quality of life of the patients. Surgery has gradually lost its importance and it is often replaced by combined chemoradiotherapy. Response to chemoradiotherapy is very variable and, at present, it is not possible to know which patients will benefit from it. Genetic assessment of head and neck squamous cell carcinomas may help clinicians to perform a well-shaped therapy, adapted on the basis of the patient's genetic features. This strategy is presently experimental, but it may become the 'standard' in the future.

First draft submitted: 24 May 2018; Accepted for publication: 31 July 2018; Published online:

26 October 2018

Keywords: accelerated $\bullet$ cyclin D1 $\bullet$ EGFR $\bullet$ genetics $\bullet$ head and neck $\bullet$ HPV $\bullet$ hyperfractionated $\bullet$ P16 $\bullet$ radiation therapy $\bullet$ radiotherapy $\bullet$ translational research

The role of radiotherapy in locally advanced squamous cell carcinoma of the head \& neck

Radiation therapy is often chosen as primary treatment for locally advanced squamous cell carcinoma of the head and neck (LA-SCCHN), mainly due to their unresectability. Moreover, upfront chemoradiation or radiotherapy alone (for unfit patients) represents the preferred options also for some subgroups of SCCHN, including hypopharyngeal, oropharyngeal and supraglottic laryngeal primitives. In the last decade, radiation therapy has become a very important treatment for SCCHN [1-4], since over the years many new techniques able to conform the maximum dose to the target have been developed. $2 \mathrm{D}$ treatments have slowly been replaced by conformal-3D techniques and, in turn, the latter have almost been substituted by intensity-modulated radiation therapy. 
Today, intensity-modulated radiation therapy and volumetric-modulated arc therapy techniques are commonly employed with or without systemic therapy in patients with LA-SCCHN, achieving good overall response rates (ORR), ranging from 75 to $90 \%$ [5-10].

Nevertheless, tumor responses to these techniques vary widely, and currently it is not possible to establish which patients will particularly benefit from radiation or chemoradiation. Of note, improving radiotherapy techniques does not always lead to a better prognosis and the possible explanation of this phenomenon lies in the genetics of SCCHN.

\section{Genetics of SCCHN}

SCCHN are a very heterogeneous group of disease, for both clinical and molecular aspects. In 2004, Chung et al. identified four distinct subtypes of SCCHN, namely basal-like, classical, mesenchymal and atypical variants, by microarray analysis of 582 genes. The so called 'basal-like variant' displayed high EGFR expression at immune-staining, associated to a well-differentiated (keratinizing) histology. The 'mesenchymal' subtype showed overexpression of genes typical of fibroblasts, including vimentin and syndecan, suggesting the activation of the epithelial-mesenchymal transition process in the cancer cells. The 'classical' variant showed high level of antioxidant enzymes, such as glutathione-S-transferase and thioredoxin reductase, which are genes highly expressed in the epithelium of strong smokers. Finally, the 'atypical' variant did not display keratinizing histology and appeared to be poorly differentiated and to express low level of EGFR [11]. This line of research has been recently resumed by Walter et al. who performed a similar analysis in patients affected by SCCHN, but, in addition, they evaluated the expression of other crucial genes, such as CCND1, TP53 and PIK3CA, and, mainly, they also assessed the status of human papillomavirus (HPV). As results, they were able to further divide the so called 'atypical subtype' into two classes, namely HPV related and not HPV related [12].

Summing up, the final result of these researches (Chung and Walter) was the discovery of several entities that differ widely among themselves. Prognosis was very different in these subtypes, as it was worse in the 'classical' and 'basal-like' variants.

We can summarize the above-described results highlighting that SCCHN are not a single entity, but they should be divided on the basis of their genetics. As an example, HPV-related SCCHN, which belongs to the 'atypical' variant described by Chung, shows typical features, such as low number of gene mutations, low EGFR expression and a wild-type status for TP53 and CyclinD1. Clinically, HPV-related tumors are characterized by good chemo- and radio-sensitivity [13,14]. On the other hand, alcohol and tobacco-related SCCHN (basal like, classical, mesenchymal and part of atypical) are more likely to be chemo- and radio-resistant; they often harbor high number of mutations, among which TPF5 and CCND1 (the gene encoding for Cyclin D1) are particularly interesting [15-18].

Basal-like variants of SCCHN are often HPV negative and are frequently related to alcohol and tobacco consumption. Interestingly, the basal-like variant is characterized by high EGFR expression, which often corresponds to EGFR gene amplification. This genome aberration has been shown to be mutually exclusive with HPV status in several studies [19-21].

In conclusion, of the above-described features is that SCCHN showing high EGFR expression are almost always not virus-related entities and, described as basal-like variant by Chung, often shows EGFR gene amplification and strong correlation with alcohol and tobacco consumption. Basal-like SCCHN variant, shows also poor prognosis in clinical trials.

\section{Genetics of SCCHN \& implications on therapy}

Given the high chemo- and radio-sensitivity of HPV-related SCCHN, locally advanced tumors may be treated with conservative strategies. Recently, Cmelack et al. have presented data of a Phase II study in which HPV-related SCCHN were treated with induction chemotherapy docetaxel, cisplatin and 5-fluorouracil (TPF) followed by a de-escalated chemoradiation regimen consisting of a total of 50 Gy associated with the anti-EGFR monoclonal antibody cetuximab. Preliminary results showed that these patients had better outcome compared with those with HPV-negative tumors who were treated with standard (70 Gy) radiotherapy [22]. In the future, the acquisition that some SCCHN are virus-related diseases can be sufficient to manage them with conservative and underpowered chemoradiotherapy, thus avoiding demolitive surgery and positively impacting not only on survival but also on quality of life of the affected patients. Nevertheless, it is important to clarify that not all HPV-positive SCCHN are also HPV-related malignancies, because the HPV is not always the 'main driver' of cancerogenesis. So, our efforts 
must be aimed at clearly dividing virus-related tumors from nonvirus-related ones, and this can be achieved by choosing the most suitable markers typical of related virus tumors (such as p16 overexpression, TP53 wild-type status and Cyclin D1 wild-type status).

On the other hand, smoke and tobacco-related SCCHN seem to be chemo- and radio-resistant, thus conservative treatments might be discouraged in these categories of patients.

However, a subgroup of HPV nonrelated SCCHN, in particular those belonging to the 'basal-like' variant, could be treated with radiation or chemoradiation, albeit they are considered radio resistant. The reason for this statement lies in the particular biological features of these tumors.

\section{EGFR \& response to radiation therapy}

EGFR is a tyrosine kinase growth factor receptor, and a member of the HER family. EGFR activation triggers a phosphorylation cascade mediated by the PI3K-PTEN-AKT, MAPK, ERK and Jak/STAT pathways, and ultimately promotes proliferation, invasion, angiogenesis, and metastatic spread [23-25]. Aberrant activation of EGFR signaling in SCCHN can be mediated by several mechanisms, including EGFR gene amplification, overexpression of EGFR and its ligands, establishment of autocrine/paracrine loops, EGFR mutation/polymorphism and transactivation by other receptor tyrosine kinases [26,27]. Importantly, EGFR overexpression correlates with poor prognosis in SCCHN, independently from the molecular mechanism of EGFR activation. The main reason explaining this phenomenon may be the accelerated tumor cell repopulation after radiation therapy. Indeed, marked EGFR overexpression leads to constitutive activation of the downstream pathway effectors, with generation of sustained proliferative ligand-mediated signals. Evidence from clinical trials demonstrated a positive correlation between the intensity of EGFR expression and the proliferation index Ki-67 in SCCHN. The repopulation effect derived from proliferation increase may counteract the effects of radiotherapy [28-30].

Several clinical trials have clearly demonstrated that high EGFR expression strongly correlates with poor prognosis and lower response rate after standard radiotherapy or chemoradiation in patients affected by LA SCCHN [31,32].

A potential strategy to circumvent this accelerated cells repopulation may be increasing the total number of radiotherapy fractions and simultaneously reduce the delivery interval, as recently suggested in a large randomized trial [33].

Moreover, several randomized controlled studies have shown that increasing the rate of dose accumulation per week leads to an increased tumor-control probability in SCCHN [34-37].

Conventional fractionation radiotherapy is generally administered delivering a dose of 44/46 Gy in 22/23 fractions upon a large volume (including the primary tumor, any involved lymph nodes and the relevant area of lymphatic drainage), followed by an additional 22/24 Gy in 11/12 fractions upon a small volume (including the primary tumor and the known nodal involvement with a margin). Thus, a total dose of 66/70 Gy in 2-Gy fractions is delivered with one fraction per day, 5 days per week, during a planned total overall treatment time of 45 days.

On the other hand, altered fractionation radiation therapy schemes concentrate more fractions in a small time interval, increasing the radiation dose administered in the time unit. Continuous accelerated hyperfractionated radiation therapy (CHART), for example, consists in the administration of 1.5 Gy per fraction, three fractions per day, with a strict $6 \mathrm{~h}$ interval between each fraction. Radiotherapy is delivered over 12 consecutive days, always starting on a Monday, treating on Saturday and Sunday, and finishing on the Friday of the second week. The large volume receives $37.5 \mathrm{~Gy}$ in 25 fractions, and the small volume receives an additional 16.5 Gy in 11 fractions, thus delivering a total dose of $54 \mathrm{~Gy}$ in 36 fractions in just 12 days to the gross tumor volume.

In a Phase III randomized trial, Soren et al. compared a standard fractionating regimen versus CHART, in patients affected by LA SCCHN. The two regimens were able to obtain similar ORR, but a subgroup analyses revealed that in patients with high EGFR expression at immunostaining $(>25 \%$ of the total cellularity in tissue samples), CHART was associated with a better response rate and survival [34].

In another Phase III trial, carried out by Bentzen $e$ t al., patients affected by LA SCCHN were randomized to receive an accelerated or a standard-fractionated adiation therapy (RT). As results, high EGFR expression showed a trend as independent determinant of overall survival, progression-free survival and locoregional recurrence rate (LRR) in the accelerated arm, although statistical significance was not reached. In addition, immunohistochemical analysis performed in pretreatment tumor biopsies revealed a beneficial role of high EGFR expression in patients assigned to CHART, compared with those receiving conventionally fractionated RT. Specifically, in a subgroup of patients showing high EGFR expression (cells with EGFR membrane staining $\geq 25 \%$ ) a significant benefit in 
3-year locoregional control rate was observed in the CHART arm compared with the conventionally fractionated RT arm. No difference between the two treatment arms was observed in the low EGFR-expressing group [35].

Eriksen et al. conducted a subgroup analysis on 803 patients enrolled in the DAHANCA 6 and 7 trials, treated with moderately accelerated radiotherapy or alternatively with conventional radiotherapy. Again, tumors with high EGFR expression responded better to moderately accelerated radiotherapy, compared with low EGFR-expressing carcinomas. Interestingly, tumors with high EGFR levels and well/moderate differentiation responded particularly to a moderate acceleration of treatment in terms of locoregional control, suggesting a potential role of other factors besides EGFR in influencing response to radiotherapy [36].

Suwinsky et al. analyzed tumor samples from 148 LA SCCHN patients treated with different radiotherapy regimens. They found that in addition to high EGFR immunohistochemical expression, high Ki-67 and absence of TP53 mutations also significantly impacted predicted benefit from accelerated treatment, in terms of ORR [37].

Taken together, the aforementioned evidence reinforces the hypothesis that altered fractionating RT regimens, including CHART, can counteract the accelerated cell repopulation after radiotherapy in patients whose tumors express high EGFR levels.

\section{Conclusion}

SCCHN belong to a heterogeneous group of malignancies, comprising different entities, which profoundly differ among each other with regard to biology, response to therapy and prognosis. Emerging data support the use of conservative (not surgical) treatments in HPV-related SCCHN, as they are particularly chemo- and radiosensitive [38-40]. Moreover, chemoradiation could be de-escalated in this category of patients, thus reducing the toxicity and ameliorating patients' quality of life. There are different ways to de-intensificate the chemoradiation treatment, and the reduction of the total dose administered to the patients is the most employed in clinical trials. Nevertheless, it is possible also to modify the concomitant systemic therapy, employing a less toxic drug to be used together with radiation therapy. Several authors have compared weekly versus tri-weekly cisplatin in concomitance with radiation therapy, but the reduction of the toxicity was counteracted by the inferiority of the weekly schedule in terms of survival [41-43]. Interestingly, Chera BS et al. have carried out a systematic meta-analysis upon patients treated with weekly versus tri-weekly cisplatin concurrent with radiotherapy, and they demonstrated that, for patients with favorable-risk human papillomavirus-associated oropharyngeal squamous cell carcinoma, a substantially decreased intensity of therapy with 60 grays of intensity-modulated radiotherapy and weekly low-dose cisplatin produced better preservation of quality of life compared with standard therapies while maintaining excellent 3-year tumor control and survival [44].

Among nonvirus-related SCCHN, which are often smoke- and alcohol-derived, we can find the so-called basal-like variant, characterized by high EGFR expression and well differentiation grade $[11,12,15]$.

These tumors can show resistance to radiotherapy, especially if a conventional fractionation regimen is employed. Translational studies have highlighted the fairly good responsiveness of this type of SCCHN to altered fractionating radiotherapy regimens, such as CHART [34-37]. In addition, high EGFR expression sustains tumor cell repopulation between radiotherapy fractions, as a consequence of the activation of several downstream intracellular effectors mediating cell proliferation and angiogenesis. Basing on these findings, reducing the interval between radiotherapy fractions may counteract the effect of EGFR pathway deregulation. In this regard, CHART (or other altered fractionating radiotherapy regimens) is a promising and relatively inexpensive treatment strategy, which could be employed more extensively in clinical practice with the only concern of acute radiation-related toxicities, identified in clinical trials. The only objection we could raise is the difficulty in coupling altered fractionating RT schemes with chemotherapy. We well know that chemoradiation represents the standard treatment in LA-SCCHN, being able to improve both ORR and survival, but cisplatin is very hard to administer in concomitance with CHART, due to their potential cumulative acute toxicity. A step forward may be to best incorporate altered fractionation RT schemes with systemic therapy (which may not be cisplatin, but also other less toxic agents).

Considering the clinical and molecular heterogeneity of SCCHN, we can envision that, in a near future, the treatment of patients affected by LA SCCHN should take into account their genetics, and not just focus on improving the radiotherapy techniques, which does not always lead to survival advantages.

\section{Future perspective}

In the near future, the aim of research should be to perform a well-shaped therapy on the basis of the genetic signature of the tumor and the genetic characteristics of the patient. Specific genic mutations may confer to the 
tumor more aggressiveness but also more sensitivity to specific therapies. On the other hand, a number of somatic genic polymorphisms (genes encoding for DNA repair enzymes for example) found in some patients, should render them more suitable for radiotherapy and/or chemotherapy using alkylating agents. The future effort of translational research should be to study the patients and tumor characteristics, and to choose a personalized therapy.

\section{Executive summary}

The role of radiotherapy in locally advanced squamous cell carcinoma of the head and neck

- This review analyzes the importance of genetic assessment of SCCHN before performing any type of treatment, in particular, the role of altered fractionation radiotherapy regimens is described.

Genetics of SCCHN

- SCCHN are very heterogeneous, and we will analyze their differencies from a genetic point of view.

Genetics of SCCHN and implications on therapy

- Genetic differences also imply different therapeutic strategies - we will describe them based on literature data.

EGFR and response to radiation therapy

- EGFR expression may strongly impact on response to some types of therapy, in particular, altered fractionation radiation therapy.

\section{Conclusion}

- The pre-therapy assessment is crucial before performing any kind of therapeutic strategy.

Future perspective

- To assess a specific therapy based on the specific tumor genetic signature.

\section{Acknowledgments}

The authors thank M Pontone and M L Marciano for their contribution in the drafting of the paper and in taking care of the editorial part.

Financial \& competing interests disclosure

Funds for this work have been provided by the Italian National Research Council of Naples. The authors have no other relevant affiliations or financial involvement with any organization or entity with a financial interest in or financial conflict with the subject matter or materials discussed in the manuscript apart from those disclosed.

No writing assistance was utilized in the production of this manuscript.

\section{Authors' contributions}

D V Scarpati retrieved the data from the literature; P Francesco wrote the paper; and M Giuliano edited the final version. All authors provided critical feedback and helped shape the research, analysis and manuscript.

\section{Open access}

This work is licensed under the Creative Commons Attribution 4.0 License. To view a copy of this license, visit http://creativecomm ons.org/licenses/by/4.0/

\section{References}

1. Jacinto JK, Co J, Mejia MB, Regala EE. The evidence on effectiveness of weekly vs triweekly cisplatin concurrent with radiotherapy in locally advanced head and neck squamous cell carcinoma (HNSCC): a systematic review and meta-analysis. Br. J. Radiol. 90(1079), 20170442 (2017)

2. Schick U, Huguet F, Pointreau Y, Pradier O. Radiotherapy for head and neck squamous cell carcinoma: state of the art and future directions. Cancer Radiother. 21(6-7), 498-504 (2017).

3. Perri F, Muto P, Aversa C et al. Integrated therapeutic approaches in head and neck cancer: the importance of multidisciplinary team management. Anticancer Agents Med. Chem. 13(6), 834-43 (2013).

4. NCCN Guidelines 2018 version. Head and neck squamous cell carcinoma. www.nccn.org/professionals/physician_gls/pdf/head-and-neck.pdf

5. van der Veen J, Nuyts S. Can intensity-modulated-radiotherapy reduce toxicity in head and neck squamous cell carcinoma? Cancers (Basel) 9(10), pii: E135 (2017).

6. Lambrecht M1, Nevens D, Nuyts S. Intensity-modulated radiotherapy vs. parotid-sparing 3D conformal radiotherapy. Effect on outcome and toxicity in locally advanced head and neck cancer. Strablenther Onkol. 189(3), 223-229 (2013). 
7. Moncharmont C, Vallard A, Guy JB, Prades JM, Rancoule C, Magné N. Real-life efficacy of volumetric modulated arc therapy in head and neck squamous cell carcinoma. Eur. Ann. Otorhinolaryngol. Head Neck Dis. 134(3), 165-169 (2017).

8. Moncharmont C, Vallard A, Mengue Ndong S et al. Real-life assessment of volumetric modulated arc therapy (VMAT) toxicity in head and neck squamous cell carcinoma (HNSCC) treatment. Acta Otolaryngol. 136(2), 181-188 (2016).

9. Thomson DJ, Ho KF, Ashcroft L et al. Dose intensified hypofractionated intensity-modulated radiotherapy with synchronous cetuximab for intermediate stage head and neck squamous cell carcinoma. Acta Oncol. 54(1), 88-98 (2015).

10. Rastogi M, Sapru S, Gupta P et al. Prospective evaluation of intensity modulated radiation therapy with simultaneous integrated boost (IMRT-SIB) in head and neck squamous cell carcinoma in patients not suitable for chemo-radiotherapy. Oral Oncol. 67, 10-16 (2017).

11. Chung CH, Parker JS, Karaca G et al. Molecular classification of head and neck squamous cell carcinomas using patterns of gene expression. Cancer Cell. 5(5), 489-500 (2004).

12. Walter $\mathrm{V}$, Yin $\mathrm{X}$, Wilkerson MD et al. Molecular subtypes in head and neck cancer exhibit distinct patterns of chromosomal gain and loss of canonical cancer genes. PLoS ONE 8(2), e56823 (2013).

13. Tornesello ML, Perri F, Buonaguro L, Ionna F, Buonaguro FM, Caponigro F. HPV-related oropharyngeal cancers: from pathogenesis to new therapeutic approaches. Cancer Lett. 351(2), 198-205 (2014).

14. Husain N, Neyaz A. Human papillomavirus associated head and neck squamous cell carcinoma: controversies and new concepts. J. Oral Biol. Craniofac. Res. 7(3), 198-205 (2017).

15. Zhou G, Liu Z, Myers JN. TP53 mutations in head and neck squamous cell carcinoma and their impact on disease progression and treatment response. J. Cell Biochem. 117(12), 2682-2692 (2016).

16. Peltonen JK, Vähäkangas KH, Helppi HM, Bloigu R, Pääkkö P, Turpeenniemi-Hujanen T. Specific TP53 mutations predict aggressive phenotype in head and neck squamous cell carcinoma: a retrospective archival study. Head Neck Oncol. 3, 20 (2011).

17. Feng Z, Guo W, Zhang C et al. CCND1 as a predictive biomarker of neoadjuvant chemotherapy in patients with locally advanced head and neck squamous cell carcinoma. PLoS ONE 6(10), e26399 (2011).

18. Perri F, Pacelli R, Della Vittoria Scarpati G et al. Radioresistance in head and neck squamous cell carcinoma: biological bases and therapeutic implications. Head Neck 37(5), 763-770 (2015).

19. Nakano T, Yamamoto H, Nakashima T et al. Molecular subclassification determined by human papillomavirus and epidermal growth factor receptor status is associated with the prognosis of oropharyngeal squamous cell carcinoma. Hum. Pathol. 50, 51-61 (2016)

20. Szabó B, Nelhubel GA, Kárpáti A et al. Clinical significance of genetic alterations and expression of epidermal growth factor receptor (EGFR) in head and neck squamous cell carcinomas. Oral Oncol. 47(6), 487-496 (2011).

21. Chau NG, Perez-Ordonez B, Zhang K et al. The association between EGFR variant III, HPV, p16, c-MET, EGFR gene copy number and response to EGFR inhibitors in patients with recurrent or metastatic squamous cell carcinoma of the head and neck. Head Neck Oncol. 3, 11 (2011).

22. Cmelak A, Li S, Marur S et al. Reduced-dose IMRT in human papillomavirus (HPV)-associated resectable oropharyngeal squa-mous carcinomas (OPSCC) after clinical complete re-sponse (cCR) to induction chemotherapy (IC). J. Clin. Oncol. 32, 5s (2014) (Suppl.; Abstr. LBA6006).

23. Maxwell SA, Sacks PG, Gutterman JU, Gallick GE. Epidermal growth factor receptor protein-tyrosine kinase activity in human cell lines established from squamous carcinomas of the head and neck. Cancer Res. 49, 1130-1137 (1989).

24. Kearsley JH, Kearsley JH, Furlong KL, Cooke RA, Waters MJ. An immunohistochemical assessment of cellular proliferation markers in head and neck squamous cell cancers. Br. J. Cancer 61, 821-827 (1990).

25. Ishitoya J, Toriyama $\mathrm{M}$, Oguchi $\mathrm{N}$ et al. Gene amplification and overexpression of EGF receptor in squamous cell carcinomas of the head and neck. Br. J. Cancer 59, 559-562 (1989).

26. Bossi P, Resteghini C, Paielli N, Licitra L, Pilotti S, Perrone F. Prognostic and predictive value of EGFR in head and neck squamous cell carcinoma. Oncotarget 7(45), 74362-74379 (2016).

27. Beck TN, Georgopoulos R, Shagisultanova EI et al. EGFR and RB1 as dual biomarkers in HPV-negative head and neck cancer. Mol. Cancer Ther. 15(10), 2486-2497 (2016).

28. Della Vittoria Scarpati G, Perri F, Pisconti $S$ et al. Concomitant cetuximab and radiation therapy: a possible promising strategy for locally advanced inoperable non-melanoma skin carcinomas. Mol. Clin. Oncol. 4(4), 467-471 (2016).

29. Pedicini P, Nappi A, Strigari L et al. Correlation between EGFR expression and accelerated proliferation during radiotherapy of head and neck squamous cell carcinoma. Radiat. Oncol. 7, 143 (2012).

30. Corvò R, Giaretti W, Sanguineti G et al. Potential doubling time in head and neck tumors treated by primary radiotherapy: preliminary evidence for a prognostic significance in local control. Int. J. Radiat. Oncol. Biol. Phys. 27(5), 1165-1172 (1993).

31. Lattanzio L, Denaro N, Vivenza D et al. Elevated basal antibody-dependent cell-mediated cytotoxicity (ADCC) and high epidermal growth factor receptor (EGFR) expression predict favourable outcome in patients with locally advanced head and neck cancer treated with cetuximab and radiotherapy. Cancer Immunol. Immunother. 66(5), 573-579 (2017). 
32. Solomon MC, Vidyasagar MS, Fernandes D et al. The prognostic implication of the expression of EGFR, p53, cyclin D1, Bcl-2 and p16 in primary locally advanced oral squamous cell carcinoma cases: a tissue microarray study. Med. Oncol. 33(12), 138 (2016).

33. Overgaard J, Hansen HS, Specht L. Five compared with six fractions per week of conventional radiotherapy of squamous-cell carcinoma of head and neck: DAHANCA 6 and 7 randomised controlled trial. Lancet 362, 933-940 (2003). [Erratum: Lancet 362:1588, 2003].

34. Chung $\mathrm{CH}$, Zhang Q, Hammond EM et al. Integrating epidermal growth factor receptor assay with clinical parameters improves risk classification for relapse and survival in head-and-neck squamous cell carcinoma. Int. J. Radiat. Oncol. Biol. Phys. 81(2), 331-338 (2011).

35. Bentzen SM, Atasoy BM, Daley FM et al. Epidermal growth factor receptor expression in pretreatment biopsies from head and neck squamous cell carcinoma as a predictive factor for a benefit from accelerated radiation therapy in a randomized controlled trial. J. Clin. Oncol. 23(24), 5560-5567 (2005).

36. Eriksen JG1, Steiniche T, Overgaard J, Danish Head and Neck Cancer study group (DAHANCA). The role of epidermal growth factor receptor and E-cadherin for the outcome of reduction in the overall treatment time of radiotherapy of supraglottic larynx squamous cell carcinoma. Acta Oncol. 44(1), 50-58 (2005).

37. Suwinski R, Jaworska M, Nikiel B et al. Predicting the effect of accelerated fractionation in postoperative radiotherapy for head and neck cancer based on molecular marker profiles: data from a randomized clinical trial. Int. J. Radiat. Oncol. Biol. Phys. 77(2), 438-446 (2010).

38. Chen AM, Felix C, Wang PC et al. Reduced-dose radiotherapy for human papillomavirus-associated squamous-cell carcinoma of the oropharynx: a single-arm, Phase II study. Lancet Oncol. 18(6), 803-811 (2017).

39. Kelly JR, Husain ZA, Burtness B. Treatment de-intensification strategies for head and neck cancer. Eur. J. Cancer 68, 125-133 (2016).

40. Maxwell JH, Grandis JR, Ferris RL. HPV-associated head and neck cancer: unique features of epidemiology and clinical management. Annu. Rev. Med. 67, 91-101 (2016).

41. Lee SY, Choi YS, Song IC et al. Comparison of standard-dose 3-weekly cisplatin and low-dose weekly cisplatin for concurrent chemoradiation of patients with locally advanced head and neck squamous cell cancer: a multicenter retrospective analysis. Medicine (Baltimore) 97(21), e10778 (2018).

42. Jacinto JK, Co J, Mejia MB, Regala EE. The evidence on effectiveness of weekly vs triweekly cisplatin concurrent with radiotherapy in locally advanced head and neck squamous cell carcinoma (HNSCC): a systematic review and meta-analysis. Br. J. Radiol. 90(1079), $20170442(2017)$.

43. Mackiewicz J, Rybarczyk-Kasiuchnicz A, Łasińska I et al. The comparison of acute toxicity in 2 treatment courses: three-weekly and weekly cisplatin treatment administered with radiotherapy in patients with head and neck squamous cell carcinoma. Medicine (Baltimore) 96(51), e9151 (2017).

44. Chera BS, Amdur RJ, Tepper JE et al. Mature results of a prospective study of deintensified chemoradiotherapy for low-risk human papillomavirus-associated oropharyngeal squamous cell carcinoma. Cancer 124(11), 2347-2354 (2018). 
